\title{
A maximum estimability criterion for design classification and selection
}

\author{
Xianggui Qu* \\ Oakland University, Rochester, MI 48309, USA
}

\begin{abstract}
A maximum estimability (maxest) criterion is proposed for design classification and selection. It is an extension and refinement of Webb's resolution criterion for general factorial designs. By using the estimability vector associated with the maxest criterion, projective properties of nonregular designs are studied from the estimability perspective. Comparisons with other criteria are also discussed.
\end{abstract}

Keywords: Fractional factorial design, generalized minimum aberration, maximum estimability, estimability vector, projection, resolution.

\section{Introduction}

Factorial designs can be broadly classified into two categories, orthogonal and nonorthogonal. There are two types of orthogonal designs, regular and nonregular. A regular design is determined by its defining contrast subgroup which consists of defining relations among the factors. It has a simple aliasing structure in which two factorial effects are either orthogonal or fully aliased (i.e., completely indistinguishable). Examples of regular designs include the widely used $2^{n-(n-k)}$ designs or more general $s^{n-(n-k)}$ designs, where $s$ is a prime or prime power, $n$ and $k$ are integers. In contrast, a nonregular design cannot be defined by a defining contrast subgroup, and as a result, some of its factorial effects are neither orthogonal nor fully aliased. Nonregular designs include Plackett-Burman designs, designs constructed from Hadamard matrices, and other symmetrical and asymmetrical orthogonal arrays (OA) as described in Hedayat et al., (1999). On the other hand, nonorthogonal designs play an important role in practical experimentation with their flexibility and economy in level combination and run size. Saturated designs in Rechtschaffner (1967), balanced arrays (Srivastava and Chopra, 1971) are nonorthogonal designs.

A fundamental issue in factorial designs is how to assign factors to the columns of a plan matrix. A plan matrix describes the experimental plan by using standard notations for factor

\footnotetext{
${ }^{*}$ Corresponding author. Tel.: +01-248-370-4029; fax: +01-248-370-4148.

E-mail address: qu@oakland.edu (Xianggui Qu)
} 
levels, such as "0" and "1" (or "low" and "high", etc.) for two levels and "0", "1" and "2" (or "low", "medium" and "high", etc.) for three levels. Box and Hunter (1961) proposed the maximum resolution criterion for regular designs. Their concept of resolution can be interpreted in two equivalent ways, namely, effect aliasing and effect estimability. From the effect-aliasing point of view, a design has resolution $R$ if no $p$-factor interactions are aliased with any other effects containing less than $R-p$ factors, where $p$ and $R$ are integers and $2 p<R$. From the estimability perspective, a design has resolution $R \geq 3$ if, when $R$ is odd, effects involving $(R-1) / 2$ or fewer factors are estimable under the assumption that those involving $(R+1) / 2$ or more factors are negligible; when $R$ is even, effects involving $(R-2) / 2$ or fewer factors are estimable under the assumption that those involving $(R+2) / 2$ or more factors are negligible. Extensions of the maximum resolution, from the effect-aliasing perspective, have been studied extensively. These include the minimum aberration (MA) criterion (Fries and Hunter, 1980), generalized minimum aberration (GMA) (Deng and Tang, 1999; Xu and Wu, 2001), minimum moment aberration (MMA) (Xu, 2003), etc.

Interpretation of the maximum resolution criterion from the estimability perspective was first generalized to nonregular as well as nonorthogonal designs by Webb (1968) and has been used to construct many useful nonorthogonal designs. However, Webb's criterion is so coarse that it is often used as a classification rule rather than a criterion for design selection. A maximum estimability (maxest) criterion is proposed for general factorial designs in Section 2. The maxest criterion is an extension and refinement of Webb's criterion. In terms of comparing regular designs of resolution IV, it is shown in Section 3 that the maxest criterion extends the MaxC2 criterion proposed in $\mathrm{Wu}$ and Hamada (2000) (hereafter abbreviated as WH). In Section 4, the maxest criterion is used to study projections of nonregular two-level as well as mixed-level designs. Comparisons with other criteria are also discussed.

\section{The maximum estimability (maxest) criterion}

Let $G_{i}=\left\{0,1, \ldots, s_{i}-1\right\}$, and $H$ be the Cartesian product of $G_{i}$, that is, $H=G_{1} \times \cdots \times G_{n}$. An asymmetrical (or mixed-level) design, denoted by $D$, of $n$ factors with levels $s_{1}, \ldots, s_{n}$ is a subset of $H$ in which each vector represents a run. $D$ is symmetrical if $s_{1}=\cdots=s_{n}$. The $n$-factor linear model which will be adopted throughout the ensuing development is defined as

$$
E(Y(x))=\sum_{u \in H} \chi_{u}(x) \beta_{u}
$$

or in matrix form

$$
E(Y)=X \beta,
$$


where $Y(x)$ is the response of treatment combination $x \in D$, and $Y$ is the vector of $Y(x)$ 's; $\beta_{u}$ is a treatment contrast (or factorial effect) and $\beta$ is the vector of $\beta_{u}$ 's; $\chi_{u}(x)$ is a normalized contrast coefficient and $X$ is the coefficient matrix or model matrix.

For $u \in H$ with $i$ nonzero elements, $\beta_{u}$ is an $i$-factor interaction. In particular, $\beta_{(0, \ldots, 0)}$ is the grand mean which has coefficient $\frac{1}{\sqrt{|H|}}$, i.e., $\chi_{(0, \ldots, 0)}(x)=\frac{1}{\sqrt{|H|}}$ for any $x \in H$, where $|H|$ is the number of elements in $H$. Two contrasts $\beta_{u}$ and $\beta_{v}$ are orthonormal if their contrast coefficients are orthonormal, i.e., $\sum_{x \in H} \chi_{u}(x) \chi_{v}(x)=\delta_{u, v}$, where $\delta_{u, v}$ is the Kronecker delta, which equals 1 if $u=v$, and 0 otherwise. In this paper, only contrasts defined by tensor products are considered. Let $\left\{\chi_{v_{i}}^{s_{i}}, v_{i} \in G_{i}\right\}$ be the orthonormal contrasts of the $i$ th factor with $s_{i}$ levels, i.e., $\sum_{x_{i} \in G_{i}} \chi_{u_{i}}^{s_{i}}\left(x_{i}\right) \chi_{v_{i}}^{s_{i}}\left(x_{i}\right)=\delta_{u_{i}, v_{i}}$ for any $u_{i}, v_{i} \in G_{i}$. Then for $u=\left(u_{1}, \ldots, u_{n}\right) \in H$ and $x=\left(x_{1}, \ldots, x_{n}\right) \in H$, define $\chi_{u}(x)=\prod_{i=1}^{n} \chi_{u_{i}}^{s_{i}}\left(x_{i}\right)$.

If there are up to $r$-factor interactions in model (1), it is called an $r$ th-order model. Thus a first-order model includes all main effects as well as the grand mean; a second-order model includes the grand mean, all main effects, and two-factor interactions (2fi's); a third-order model includes the grand mean, all main effects, 2fi's and three-factor interactions (3fi's); and so on. An effect in model (1) is said to be estimable if it has a linear unbiased estimator. The importance of estimability is that there exists a unique best linear unbiased estimator if the effect is estimable. Now we are ready to introduce the maxest criterion.

Definition 1. For a design $D$ of $n$ factors with $s_{1}, \cdots, s_{n}$ levels, let $n_{k j}(D)$ be the number of estimable $k$-factor interactions in the $j$ th-order model, $k \leq j, k, j=1,2, \ldots, n$, and $f_{k j}(D)=n_{k j}(D) / \sum_{i_{1}<i_{2}<\cdots<i_{k}}\left(s_{i_{1}}-1\right) \cdots\left(s_{i_{k}}-1\right)$, where $i_{1}, \cdots, i_{k} \in\{1,2, \cdots, n\}$. The estimability vector (hereafter abbreviated as $\mathrm{EV}$ ) of $D$ is defined as $\mathrm{EV}(\mathrm{D})=\left(f_{11}, f_{12}, f_{22}, f_{13}, f_{23}, f_{33}\right.$, $\left.\cdots, f_{1 n}, f_{2 n} \cdots, f_{n n}\right)$. The maxest criterion sequentially maximizes the components of EV(D). Let $f_{l}(D)$ be the $l$ th entry of $\operatorname{EV}(\mathrm{D}), l=1,2, \ldots, n(n+1) / 2$. For any two designs, $D_{1}$ and $D_{2}$, let $l_{0}$ be the smallest integer such that $f_{l_{0}}\left(D_{1}\right) \neq f_{l_{0}}\left(D_{2}\right)$. If $f_{l_{0}}\left(D_{1}\right)>f_{l_{0}}\left(D_{2}\right)$, then $D_{1}$ has higher estimability than $D_{2} . D_{1}$ is the maxest design if no designs have higher estimability than it.

The maxest criterion extends and refines Webb's resolution criterion. According to Webb, $f_{11}=1$ for a design of resolution III; $f_{11}=1$ and $f_{12}=1$ for a design of resolution IV; $f_{11}=1, f_{12}=1$ and $f_{22}=1$ for a design of resolution $\mathrm{V}$. Note that Webb's criterion is not able to differentiate designs of the same resolution while the maxest criterion can further classify resolution III, IV or V designs by the numbers of estimable main effects, 2fi's, and so on. Another advantage of the maxest criterion over other criteria is that the component order of the EV can be defined by users. For example, control-by-noise interactions are more important than main effects of noise factors in robust parameter design. The effect ordering principle proposed by $\mathrm{Wu}$ 
and Zhu (2003) can be achieved by placing control-by-noise interactions ahead of main effects of noise factors in the EV.

Wu and Chen (1992) proposed the concept of clear effects for regular designs, which states that a main effect or 2fi is clear if it is not aliased with any other main effects or 2fi's (hence it is estimable in the second-order model) and is strongly clear if it is not aliased with any 3fi's as well (therefore, it is estimable in the third-order model). Clear effects in Definition 2 extend Wu-Chen's definition to nonregular as well as nonorthogonal designs.

Definition 2. A main effect is eligible if it is estimable in the first-order model. A main effect or $2 \mathrm{fi}$ is clear if it is estimable in the second-order model and is strongly clear if it is estimable in the third-order model.

For simplicity, only main effects and 2fi's are considered in up to the third-order model in this paper. That is, $\mathrm{EV}=\left(f_{11}, f_{12}, f_{22}, f_{13}, f_{23}\right)$. Higher-order effects and models can be studied similarly. Since the elements of EV are relative frequencies of eligible main effects, clear main effects and 2fi's, and strongly clear main effects and 2fi's, designs with $\mathrm{EV}=(1,1,1,1,1)$ are maxest design. Recall that two designs are isomorphic if one can be obtained from the other by relabelling factors, reordering runs or switching the levels of factors. It is obvious that two isomorphic designs have the same $\mathrm{EV}$.

Generally, it is not easy to check the estimability of factorial effects. Milliken (1971) proposed a simple approach to check the estimability of linear combinations of parameters in a linear model. According to Milliken's procedure, only one number needs to be computed and compared in order to check the estimability of an effect. That is, an effect is estimable if and only if $\operatorname{rank}(X)=\operatorname{rank}\left(X^{\star}\right)+1$, where $X$ is the model matrix and $X^{\star}$ is obtained from $X$ by deleting the coefficient vector of the effect. Thus a necessary and sufficient condition for an effect to be estimable is that its coefficient vector is linearly independent of others in the model matrix.

The maxest criterion is different from the GMA criterion in $\mathrm{Xu}$ and $\mathrm{Wu}$ (2001). The former concerns the estimability of main effects and 2fi's and maximizes the number of clear effects. The latter minimizes the aliasing of high-order effects to low-order effects. Generally, as shown in Example 2, a GMA design is not necessarily a maxest design. It has been shown that the GMA criterion is independent of coding systems. Example 1 shows that the maxest criterion is coding-dependent unless all the factors appear at two levels. For a fixed design, different coding systems result in different EV's.

Example 1. Consider a three-level design of four factors, $A, B, C$, and $D$ with a defining relation $D=A B C$. If all the factors are quantitative, there are two popular coding systems, the 
orthogonal components and the linear-quadratic system (WH, Chapter 5). It can be shown that $\mathrm{EV}=(1,1,12 / 24,0,0)$ under the orthogonal components coding while $\mathrm{EV}=(1,1,0,0,0)$ for the linear-quadratic system.

Example 2. There are two 20-run orthogonal arrays in Table 2. One is the six-factor GMA design (Sun et al., 2001) and the other is a projection with columns 1, 2, 3, 6, 8, and 17 of the 20-run $\mathrm{P}$ design in Appendix A. It can be shown that the GMA design has $\mathrm{EV}=(1,0,0,0,0)$ and the projection has $\mathrm{EV}=(1,1,3 / 15,0,0)$. That is, the GMA design has no clear effects while all the main effects and three 2fi's are clear in the projection.

Table 2

20-run orthogonal arrays of six factors

\begin{tabular}{ccccccccccccc}
\hline Run & \multicolumn{1}{c}{ GMA } & \multicolumn{11}{c}{ Projection } \\
\hline 1 & 1 & 1 & 1 & 0 & 1 & 1 & 0 & 0 & 0 & 0 & 0 & 1 \\
2 & 1 & 1 & 1 & 0 & 0 & 1 & 0 & 1 & 1 & 0 & 1 & 1 \\
3 & 1 & 1 & 0 & 1 & 1 & 0 & 1 & 0 & 1 & 1 & 1 & 0 \\
4 & 1 & 1 & 0 & 1 & 0 & 1 & 1 & 1 & 0 & 1 & 0 & 1 \\
5 & 1 & 1 & 0 & 0 & 0 & 0 & 1 & 1 & 1 & 1 & 1 & 0 \\
6 & 1 & 0 & 1 & 1 & 1 & 0 & 0 & 0 & 0 & 1 & 1 & 1 \\
7 & 1 & 0 & 1 & 1 & 0 & 1 & 0 & 1 & 1 & 1 & 0 & 1 \\
8 & 1 & 0 & 1 & 0 & 0 & 0 & 1 & 0 & 1 & 0 & 0 & 1 \\
9 & 1 & 0 & 0 & 1 & 1 & 1 & 1 & 1 & 0 & 0 & 1 & 1 \\
10 & 1 & 0 & 0 & 0 & 1 & 0 & 1 & 1 & 1 & 0 & 0 & 0 \\
11 & 0 & 1 & 1 & 1 & 1 & 0 & 1 & 0 & 0 & 1 & 0 & 0 \\
12 & 0 & 1 & 1 & 1 & 0 & 1 & 1 & 1 & 0 & 0 & 0 & 0 \\
13 & 0 & 1 & 1 & 0 & 1 & 0 & 0 & 0 & 0 & 0 & 0 & 0 \\
14 & 0 & 1 & 0 & 1 & 0 & 0 & 1 & 0 & 0 & 1 & 1 & 1 \\
15 & 0 & 1 & 0 & 0 & 1 & 1 & 0 & 0 & 1 & 0 & 1 & 0 \\
16 & 0 & 0 & 1 & 1 & 0 & 0 & 0 & 1 & 1 & 1 & 0 & 1 \\
17 & 0 & 0 & 1 & 0 & 1 & 1 & 0 & 1 & 0 & 0 & 1 & 0 \\
18 & 0 & 0 & 0 & 1 & 1 & 1 & 0 & 1 & 0 & 1 & 1 & 0 \\
19 & 0 & 0 & 0 & 0 & 0 & 1 & 1 & 0 & 1 & 0 & 1 & 1 \\
20 & 0 & 0 & 0 & 0 & 0 & 0 & 0 & 0 & 1 & 1 & 0 & 0 \\
\hline
\end{tabular}

\section{The maxest criterion for regular designs}

In this section the maxest criterion is studied for regular two-level designs. As suggested by WH, designs of three-level factors or higher could be treated as either nonregular or regular depending on the coding systems. Let $D$ be a $2^{n-(n-k)}$ design. Then $D$ is completely determined by its defining contrast subgroup. The following result is self-explanatory.

Theorem 1. If $D$ is a design of resolution III or higher, $k_{12}$ denotes the number of distinct letters in three-letter words, and $k_{13}$ is the number of distinct letters in three- or four-letter words in the defining contrast subgroup. Let $k_{22}$ be the number of distinct pairs in three- or four-letter words and $k_{23}$ be the number of distinct pairs in three-, four-or five-letter words. Then

$$
E V(D)=\left(1,1-k_{12} / n, 1-2 k_{22} / n(n-1), 1-k_{13} / n, 1-2 k_{23} / n(n-1)\right) .
$$


Let $M(k)$ be the maximum number of factors that can be accommodated in a $2^{n-(n-k)}$ design of resolution V or higher. Chen and Hedayat (1998) studied the existence of clear 2fi's in $2^{n-(n-k)}$ designs. Tang, Ma, Ingram, and Wang (2000) gave the upper bound number of clear 2fi's in a resolution IV design. These results are summarized in Theorem 2 using the current terminology.

Theorem 2. Let $D$ be a maxest $2^{n-(n-k)}$ design. Then

(i) for $n \leq M(k), E V(D)=\left(1,1,1,1, f_{23}\right)$, where $0 \leq f_{23} \leq 1$,

(ii) for $M(k)<n \leq 2^{k-2}+1, E V(D)=\left(1,1, \alpha, f_{13}, f_{23}\right)$, where $0 \leq f_{13} \leq 1$ and

$$
f_{23} \leq \alpha \leq\left\{\begin{array}{ll}
\min \left(1,\left(2^{k+1}-4 n-2\right) / n(n-3)\right) & \text { if } n \text { is odd } \\
\min \left(1,\left(2^{k+1}-4 n\right) /(n-1)(n-2)\right) & \text { if } n \text { is even }
\end{array},\right.
$$

(iii) for $2^{k-2}+1<n \leq 2^{k-1}, E V(D)=(1,1,0,0,0)$,

(iv) for $2^{k-1}<n \leq 2^{k}-1, E V(D)=(1,0,0,0,0)$.

For $n \leq M(k), f_{23}$ in Theorem 2 can be 0,1 or strictly between 0 and 1 . For example, when $k=4, M(k)=5, f_{23}=0$ in the $2_{V}^{5-(5-4)}$ design with $5=1234$. When $k=5, M(k)=6$, $f_{23}=1$ in the $2_{V I}^{6-(6-5)}$ design with $6=12345$. When $k=6, M(k)=8, f_{23}=\frac{9}{28}$ in the $2_{V}^{8-(8-6)}$ design with $7=1234$ and $8=1256$. According to (iv) of Theorem 2 , no $2^{n-(n-4)}, 2^{n-(n-5)}$, and $2^{n-(n-6)}$ designs have any clear main effects or 2fi's for $8<n \leq 15,16<n \leq 31,32<n \leq 63$, respectively.

For designs of resolution IV, the GMA criterion is equivalent to the MA criterion which minimizes the number of four-letter words in the defining contrast subgroup. However, minimizing the number of four-letter words doesn't necessarily minimize the number of 2fi's being aliased or maximize the number of clear 2fi's. As a supplement to the MA criterion, WH proposed a MaxC2 criterion to select $2_{I V}^{n-(n-k)}$ designs, i.e., among all designs of resolution IV, those with the maximum number of clear 2fi's are the best. It is easy to see that the maxest criterion includes the MaxC2 criterion as a special case when designs of resolution IV are compared.

\section{Projections of nonregular designs}

Traditionally, nonregular designs are mainly used for screening because of the complex aliasing structure among their effects. A paradigm shift was first suggested by Hamada and Wu (1992), who showed that for designed experiments with complex aliasing, it is possible to estimate some interactions. Since then much work (Wang and Wu, 1995; Lin and Draper, 1995; Deng, Li and Tang, 2000, etc.) has been done on projection properties of nonregular designs. All these studies reveal that nonregular designs enjoy better projection properties, which makes it possible to do screening and optimization simultaneously (Cheng and Wu, 2001). 
Projections of 16-run Hall's designs, 20-run designs from Hadamard matrices will be studied using the EV associated with the maxest criterion in Sections 4.1 and 4.2. Mixed-level design $O A\left(36,3^{12} 2^{11}\right)$ is analyzed in Section 4.3. Comparisons of results with Lin-Draper's (LD), WangWu's (WW), and Deng-Li-Tang's (DLT) will be discussed as well.

\subsection{Projections of 16-run Hall's designs}

Hall's designs of 16 runs are the ones in which both full aliasing and partial aliasing occur. There are five non-isomorphic Hall's designs, labelled as H16I, H16II, H16III, H16IV and H16V. In particular, H16I is the regular $2_{I I I}^{15-(15-4)}$ design. Lists of H16II to H16V can be found in Appendix 7B of WH.

Classes of projections in H16II to H16V and their EV's are summarized in Table 3, where M16- $n . k$ is the $k$ th class when any of the four designs are projected onto $n$ factors. These classes are arranged in an ascending order of estimability. For example, M16-5.1 has no clear effects and M16-5.2 has one clear 2fi. Therefore, M16-5.2 has higher estimability than M16-5.1. For each design, the percentage of projections of M16- $n . k$ is listed. For instance, $7.2 \%$ of 3003 projections onto five factors in H16II are of M16-5.9. This is actually the maxest projection among all 5dimensional projections. None of 5-dimensional projections in H16IV and H16V are of M16-5.9. Projections onto 9 or more factors are not listed because they have no clear main effects or 2fi's. That is, $\mathrm{EV}=(1,0,0,0,0)$. The rest of this section is devoted to discussing Table 3.

Designs H16II to H16V have the same five classes of projections onto four factors and H16IV and H16V have the same frequencies as well. All the main effects and 2fi's are strongly clear in projections of M16-4.5 (the maxest four-dimensional projections). LD pointed out that designs H16II, H16III, H16IV and H16V have 4, 4, 3, 5 types of geometric projections, respectively. The five projections, denoted by LD4.a to LD4.e, in LD (page 116) and their EV's are given in Table 4. Recall that isomorphic designs have the same EV, 1, 1, and 2, types of 4-dimensional projections in H16II, H16III, and H16IV are overlooked by LD.

Designs H16II and H16III have the same 9 classes of projections onto five factors, but their frequencies are different. H16IV and H16V have the same 8 classes and frequencies. All the main effects are strongly clear and all 2fi's are clear in projections of M16-5.9 (the maxest projections). In contrast, H16II, H16III, H16IV and H16V have 6, 6, 4, 7 geometric classes in LD, respectively. The eight projections, denoted by LD5.a to LD5.h, in LD (page 117) and their EV's are given in Table 4. Similarly, 3, 3, 4, and 1 classes of 5-dimensional projections in H16II, H16III, H16IV, and $\mathrm{H} 16 \mathrm{~V}$ are overlooked by LD.

According to the maxest criterion, H16II is the best. It has the largest proportion of maxest 
Table 3

Projections of H16II, H16III, H16IV, and H16V

\begin{tabular}{|c|c|c|c|c|c|}
\hline Class & EV & H16II & H16III & H16IV & $\mathrm{H} 16 \mathrm{~V}$ \\
\hline$\overline{\mathrm{M} 16-3.1}$ & $(1,0,0,0,0)$ & 4.18 & 2.42 & 1.54 & 1.54 \\
\hline M16-3.2 & $(1,1,1,1,1)$ & 95.82 & 97.58 & 98.46 & 98.46 \\
\hline M16-4.1 & $(1,1 / 4,3 / 6,1 / 4,0)$ & 16.70 & 9.67 & 6.15 & 6.15 \\
\hline M16-4.2 & $(1,1,0,0,0)$ & 4.18 & 2.42 & 1.54 & 1.54 \\
\hline M16-4.3 & $(1,1,1,1 / 4,0)$ & 14.07 & 21.10 & 24.62 & 24.62 \\
\hline M16-4.4 & $(1,1,1,2 / 4,1 / 6)$ & 21.10 & 31.65 & 36.92 & 36.92 \\
\hline M16-4.5 & $(1,1,1,1,1)$ & 43.96 & 35.16 & 30.77 & 30.77 \\
\hline M16-5.1 & $(1,0,0,0,0)$ & 3.30 & 14.90 & 23.08 & 23.08 \\
\hline M16-5.2 & $(1,0,1 / 10,0,0)$ & 9.59 & 7.99 & 5.59 & 5.59 \\
\hline M16-5.3 & $(1,1 / 5,0,0,0)$ & 4.80 & 4.00 & 2.80 & 2.80 \\
\hline M16-5.4 & $(1,2 / 5,7 / 10,2 / 5,1 / 10)$ & 25.57 & 13.59 & 8.39 & 8.39 \\
\hline M16-5.5 & $(1,3 / 5,7 / 10,0,0)$ & 19.18 & 22.38 & 22.38 & 22.38 \\
\hline M16-5.6 & $(1,4 / 5,6 / 10,0,0)$ & 9.58 & 11.19 & 11.19 & 11.19 \\
\hline M16-5.7 & $(1,1,4 / 10,1 / 5,4 / 10)$ & 12.79 & 6.79 & 4.20 & 4.20 \\
\hline M16-5.8 & $(1,1,1,0,0)$ & 12.79 & 19.18 & 22.38 & 22.38 \\
\hline M16-5.9 & $(1,1,1,1,0)$ & 7.20 & 0.80 & 0.00 & 0.00 \\
\hline M16-6.1 & $(1,0,0,0,0)$ & 6.01 & 14.13 & 14.83 & 19.72 \\
\hline M16-6.2 & $(1,0,1 / 15,0,0)$ & 0.00 & 19.18 & 33.57 & 33.57 \\
\hline M16-6.3 & $(1,0,3 / 15,0,0)$ & 12.47 & 8.15 & 6.71 & 3.36 \\
\hline M16-6.4 & $(1,0,4 / 15,0,0)$ & 11.51 & 9.59 & 6.71 & 6.71 \\
\hline M16-6.5 & $(1,0,9 / 15,0,0)$ & 1.44 & 0.32 & 0.00 & 0.00 \\
\hline M16-6.6 & $(1,1 / 6,0,0,0)$ & 0.00 & 7.67 & 13.43 & 13.43 \\
\hline M16-6.7 & $(1,1 / 6,2 / 15,0,0)$ & 5.75 & 2.88 & 0.00 & 0.00 \\
\hline M16-6.8 & $(1,1 / 6,3 / 15,0,0)$ & 23.02 & 19.18 & 13.43 & 13.43 \\
\hline M16-6.9 & $(1,1 / 6,5 / 15,1 / 6,0)$ & 14.39 & 5.51 & 2.52 & 3.36 \\
\hline M16-6.10 & $(1,2 / 6,1 / 15,0,0)$ & 8.63 & 5.75 & 5.03 & 2.52 \\
\hline M16-6.11 & $(1,3 / 6,6 / 15,0,0)$ & 8.63 & 1.92 & 0.00 & 0.00 \\
\hline M16-6.12 & $(1,4 / 6,0,0,0)$ & 5.75 & 4.80 & 3.36 & 3.36 \\
\hline M16-6.13 & $(1,1,0,0,0)$ & 2.40 & 0.92 & 0.42 & 0.52 \\
\hline M16-7.1 & $(1,0,0,0,0)$ & 24.66 & 46.54 & 59.97 & 60.71 \\
\hline M16-7.2 & $(1,0,1 / 21,0,0)$ & 19.09 & 30.96 & 22.19 & 28.72 \\
\hline M16-7.3 & $(1,0,2 / 21,0,0)$ & 2.24 & 0.37 & 0.00 & 0.00 \\
\hline M16-7.4 & $(1,0,3 / 21,0,0)$ & 8.95 & 4.48 & 5.22 & 0.00 \\
\hline M16-7.5 & $(1,0,4 / 21,0,0)$ & 5.59 & 0.75 & 0.00 & 0.00 \\
\hline M16-7.6 & $(1,1 / 7,0,0,0)$ & 9.70 & 10.32 & 7.40 & 9.57 \\
\hline M16-7.7 & $(1,1 / 7,1 / 21,0,0)$ & 2.24 & 0.37 & 0.00 & 0.00 \\
\hline M16-7.8 & $(1,1 / 7,2 / 21,0,0)$ & 6.71 & 3.36 & 3.90 & 0.00 \\
\hline M16-7.9 & $(1,1 / 7,6 / 21,1 / 7,0)$ & 2.61 & 0.87 & 0.00 & 0.87 \\
\hline M16-7.10 & $(1,2 / 7,2 / 21,0,0)$ & 5.59 & 0.75 & 0.00 & 0.00 \\
\hline M16-7.11 & $(1,3 / 7,0,0,0)$ & 2.24 & 1.12 & 1.31 & 0.00 \\
\hline M16-7.12 & $(1,1,0,0,0)$ & 0.37 & 0.12 & 0.00 & 0.12 \\
\hline M16-8.1 & $(1,0,0,0,0)$ & 71.05 & 85.31 & 89.09 & 92.03 \\
\hline M16-8.2 & $(1,0,1 / 28,0,0)$ & 15.66 & 9.57 & 6.96 & 6.09 \\
\hline M16-8.3 & $(1,0,2 / 28,0,0)$ & 5.59 & 1.49 & 1.31 & 0.00 \\
\hline M16-8.4 & $(1,1 / 8,0,0,0)$ & 4.48 & 2.74 & 1.99 & 1.74 \\
\hline M16-8.5 & $(1,1 / 8,2 / 28,0,0)$ & 1.86 & 0.50 & 0.44 & 0.00 \\
\hline M16-8.6 & $(1,1 / 8,7 / 28,1 / 8,0)$ & 0.37 & 0.12 & 0.00 & 0.12 \\
\hline M16-8.7 & $(1,2 / 8,0,0,0)$ & 0.93 & 0.25 & 0.22 & 0.00 \\
\hline M16-8.8 & $(1,1,0,0,0)$ & 0.05 & 0.02 & 0.00 & 0.02 \\
\hline
\end{tabular}


Table 4

Geometric projections onto four and five factors

\begin{tabular}{llllllll}
\hline Design & EV & Design & EV & Design & EV & Design & EV \\
\hline LD4.a & $\left(1, \frac{1}{4}, \frac{3}{6}, \frac{1}{4}, 0\right)$ & LD4.b & $(1,1,0,0,0)$ & LD4.c & $(1,1,1,1,1)$ & LD4.d & $\left(1,1,1, \frac{1}{4}, 0\right)$ \\
LD4.e & $\left(1,1,1, \frac{2}{4}, \frac{1}{6}\right)$ & & & & & & \\
LD5.a & $(1,0,0,0,0)$ & LD5.b & $\left(1, \frac{2}{5}, \frac{7}{10}, \frac{2}{5}, \frac{1}{10}\right)$ & LD5.c & $\left(1,1, \frac{4}{10}, \frac{1}{5}, \frac{4}{10}\right)$ & LD5.d & $(1,1,1,1,0)$ \\
LD5.e & $\left(1,0, \frac{1}{10}, 0,0\right)$ & LD5.f & $(1,1,1,0,0)$ & LD5.g & $(1,0,0,0,0)$ & LD5.h & $\left(1, \frac{1}{5}, 0,0,0\right)$ \\
\hline
\end{tabular}

projections and the smallest proportion of projections with minimum estimability at each dimension except three (where H16IV and H16V are the best). Design H16III is the second best, H16V the third and H16IV the fourth. In terms of GMA, H16III is favored because most of the top 10 GMA projections in DLT are from H16III.

\subsection{Projections of 20-run designs from Hadamard matrices}

There are three non-isomorphic Hadamard matrices of order 20, commonly called N, P and Q. In particular, Q is equivalent to the 20-run Plackett-Burman design (WH, Appendix 7A.2). Designs $\mathrm{N}$ and $\mathrm{P}$ are given in Appendix A. Let M20-n. $k$ denote the $k$ th class of projections onto $n$ factors for designs N, P and Q. A summary of projections onto 4 to 7 factors is given in Table 5 . Neither projections onto 3 factors nor projections onto 8 or more factors are listed because there is only one class in each case. All the main effects and 2fi's are strongly clear in 3-dimensional projections, i.e., $\mathrm{EV}=(1,1,1,1,1)$. Projections onto 8 more factors have no clear main effects or 2fi's, i.e., $\mathrm{EV}=(1,0,0,0,0)$. The rest of this section will discuss results in Table 5.

Designs N, P and Q have the same 3 classes and frequencies of projections onto four factors. All 4-dimensional projections have clear main effects and 2fi's. The maxest projections are of M20-4.3 and have all the main effects and 2fi's being strongly clear. There are three projections in LD, 20-4.1, 20-4.2, and 20-4.3. Their EV's are $(1,1,1,0,0),(1,1,1,1 / 4,0)$, and $(1,1,1,1,1)$, respectively.

For 5-dimensional projections, designs $\mathrm{N}, \mathrm{P}$ and $\mathrm{Q}$ have the same 5 classes but different frequencies. All the main effects and 2fi's are clear, but are not strongly clear in projections of M20-5.5 (the maxest projections). LD showed that there are 10 non-isomorphic projections, labelled by 20-5.1 to 20-5.10. These labels do not indicate their relative ranks in terms of any design criterion. The maxest and GMA ranks of these 10 projections as well as their EV's are given in Table 6 where designs with the smallest rank is the best in terms of maxest or GMA. Again, the maxest and GMA ranks are different. For example, 20-5.8 is better than 20-5.10 in terms of GMA, but is worse in terms of maxest. This is not surprising because the two criteria 
are defined from different perspectives. However, most of the maxest ranks are consistent with the GMA ranks. That is, designs with more estimability generally have less aberration.

Designs $\mathrm{P}$ and $\mathrm{N}$ have the same 11 classes of 6-dimensional projections but different frequencies and $\mathrm{Q}$ has two fewer classes than $\mathrm{P}$ or $\mathrm{N}$. All the main effects as well as three 2 fi's are clear in projections of M20-6.11 (the maxest projections). Design Q has no projections of M20-6.11. On the other hand, there are 59, 56 and 50 non-isomorphic projections in WW. Thus, classification in this paper is coarser than WW's.

According to the maxest criterion, $\mathrm{P}$ is the best. It has the largest proportion of maxest projections and the smallest proportion of projections with minimum estimability at each dimension. Design $\mathrm{N}$ is the second best, and $\mathrm{Q}$ is the third. The GMA criterion in DLT favors $\mathrm{N}$ because almost all the top 10 projections are from $\mathrm{N}$. WW identifies $\mathrm{Q}$ as the best in terms of hidden projection properties because Q does not have the "worst" projections.

\subsection{Projections $O A\left(36,3^{12} 2^{11}\right)$}

Recall that if all the factors are quantitative, there are two popular coding systems in threelevel designs, the orthogonal components and the linear-quadratic system. Since the maxest criterion is coding-dependent, projection properties of three-level designs will be studied under a specific coding system. The difference in using two coding systems has been elaborated in Example 1. Even though the orthogonal components coding is commonly used in practice and computationally easier to handle, its contrasts are difficult to be interpreted in data analysis. On the other hand, contrasts in the linear-quadratic system have better interpretations (WH, Section 5.6). Therefore, the linear-quadratic system is favored here to exploit the projection properties of three-level designs. Results of $O A\left(36,3^{12} 2^{11}\right)$ are briefly discussed below and details are available from the author upon request. For simplicity, the strongly clear property of factorial effects will not be considered. That is, EV has three elements in the following discussion.

Projective properties of $O A\left(36,3^{12} 2^{11}\right.$ ) (WH, Table 7C.7) are complicated by the presence of two-level factors. Only projections including both two-level and three-level factors are considered here. Generally, in order to find all classes of projections onto $n_{3}$ three-level factors and $n_{2}$ two-level factors of $O A\left(36,3^{12} 2^{11}\right)$, it is necessary to enumerate all $\left(\begin{array}{l}12 \\ n_{3}\end{array}\right)\left(\begin{array}{l}11 \\ n_{2}\end{array}\right)$ projections. This is computationally intensive with regard to GMA and MMA, but is much easier for the maxest criterion. It has been verified that no projections onto five (six) or more factors have any clear effects in the two-level (three-level) part of $O A\left(36,3^{12} 2^{11}\right)$. Therefore, only $n_{2} \leq 4$ and $n_{3} \leq 5$ need to be considered.

Table 7 lists the numbers of projection classes based on the maxest criterion, EV's of the 
Table 5

Projections of $\mathrm{Q}, \mathrm{P}$ and $\mathrm{N}$

\begin{tabular}{|c|c|c|c|c|c|c|c|}
\hline \multirow[t]{2}{*}{ Class } & \multirow[t]{2}{*}{$\mathrm{EV}$} & \multicolumn{2}{|l|}{$\mathrm{Q}$} & \multicolumn{2}{|l|}{$\mathrm{P}$} & \multicolumn{2}{|l|}{$\mathrm{N}$} \\
\hline & & $\%$ & Columns & $\%$ & Columns & $\%$ & Columns \\
\hline M20-4.1 & $(1,1,1,0,0)$ & 5.88 & $\{1,2,3,16\}$ & 5.88 & $\{1,2,3,16\}$ & 5.88 & $\{1,2,3,16\}$ \\
\hline M20-4.2 & $(1,1,1,1 / 4,0)$ & 23.53 & $\{1,2,3,6\}$ & 23.53 & $\{1,2,3,6\}$ & 23.53 & $\{1,2,3,6\}$ \\
\hline M20-4.3 & $(1,1,1,1,1)$ & 70.59 & $\{1,2,3,4\}$ & 70.59 & $\{1,2,3,4\}$ & 70.59 & $\{1,2,3,4\}$ \\
\hline M20-5.1 & $(1,0,0,0,0)$ & 1.47 & $\{1,2,3,6,9\}$ & 1.86 & $\{1,2,5,6,7\}$ & 1.65 & $\{1,2,4,5,6\}$ \\
\hline M20-5.2 & $(1,1 / 5,2 / 10,0,0)$ & 13.24 & $\{1,2,3,4,11\}$ & 8.98 & $\{1,2,3,4,15\}$ & 11.66 & $\{1,2,3,4,5\}$ \\
\hline M20-5.3 & $(1,1 / 5,4 / 10,0,0)$ & 5.88 & $\{1,2,3,4,15\}$ & 4.33 & $\{1,2,3,5,17\}$ & 5.37 & $\{1,2,4,7,10\}$ \\
\hline M20-5.4 & $(1,2 / 5,3 / 10,0,0)$ & 11.76 & $\{1,2,3,4,6\}$ & 8.67 & $\{1,2,3,5,8\}$ & 10.73 & $\{1,2,4,5,18\}$ \\
\hline M20-5.5 & $(1,1,1,0,0)$ & 67.65 & $\{1,2,3,4,5\}$ & 76.16 & $\{1,2,3,4,5\}$ & 70.59 & $\{1,2,3,4,8\}$ \\
\hline M20-6.1 & $(1,0,0,0,0)$ & 52.94 & $\{1,2,3,4,5,8\}$ & 43.03 & $\{1,2,3,4,5,11\}$ & 48.4 & $\{1,2,3,4,5,6\}$ \\
\hline M20-6.2 & $(1,0,1 / 15,0,0)$ & 18.91 & $\{1,2,3,4,5,6\}$ & 17.25 & $\{1,2,3,4,5,6\}$ & 18.58 & $\{1,2,4,5,8,14\}$ \\
\hline M20-6.3 & $(1,0,2 / 15,0,0)$ & 5.04 & $\{1,2,3,4,7,10\}$ & 9.55 & $\{1,2,3,4,6,11\}$ & 7.08 & $\{1,2,4,5,6,12\}$ \\
\hline M20-6.4 & $(1,0,3 / 15,0,0)$ & 6.09 & $\{1,2,3,4,5,7\}$ & 3.18 & $\{1,2,3,6,11,18\}$ & 5.19 & $\{1,2,3,4,8,17\}$ \\
\hline M20-6.5 & $(1,1 / 6,0,0,0)$ & 7.56 & $\{1,2,3,4,5,15\}$ & 6.9 & $\{1,2,3,4,6,7\}$ & 7.43 & $\{1,2,4,5,8,9\}$ \\
\hline M20-6.6 & $(1,1 / 6,1 / 15,0,0)$ & 2.52 & $\{1,2,3,5,6,15\}$ & 4.78 & $\{1,2,3,4,5,10\}$ & 3.54 & $\{1,2,4,5,6,8\}$ \\
\hline M20-6.7 & $(1,1 / 6,2 / 15,0,0)$ & 1.26 & $\{1,2,3,7,9,17\}$ & 6.37 & $\{1,2,3,5,6,10\}$ & 2.83 & $\{1,2,3,4,8,12\}$ \\
\hline M20-6.8 & $(1,2 / 6,0,0,0)$ & 1.26 & $\{1,2,3,4,16,17\}$ & 2.39 & $\{1,2,3,4,10,15\}$ & 1.77 & $\{1,2,4,5,12,14\}$ \\
\hline M20-6.9 & $(1,2 / 6,1 / 15,0,0)$ & 4.41 & $\{1,2,3,4,5,13\}$ & 1.59 & $\{1,2,3,9,10,17\}$ & 3.54 & $\{1,2,4,5,8,10\}$ \\
\hline M20-6.10 & $(1,2 / 6,7 / 15,0,0)$ & 0.00 & NA & 4.25 & $\{1,2,3,5,6,16\}$ & 1.42 & $\{1,2,3,4,5,8\}$ \\
\hline M20-6.11 & $(1,1,3 / 15,0,0)$ & 0.00 & NA & 0.71 & $\{1,2,3,6,8,17\}$ & 0.24 & $\{4,5,6,7,8,10\}$ \\
\hline M20-7.1 & $(1,0,0,0,0)$ & 100.00 & $\{1,2,3,4,5,6,7\}$ & 96.00 & $\{1,2,3,4,5,6,7\}$ & 98.48 & $\{1,2,3,4,5,6,7\}$ \\
\hline M20-7.2 & $(1,0,1 / 21,0,0)$ & 0.00 & NA & 2.57 & $\{1,2,3,5,6,7,16\}$ & 1.14 & $\{1,2,3,5,6,7,8\}$ \\
\hline M20-7.3 & $(1,0,2 / 21,0,0)$ & 0.00 & NA & 0.29 & $\{1,2,3,5,6,8,10\}$ & 0.00 & NA \\
\hline M20-7.4 & $(1,1 / 7,0,0,0)$ & 0.00 & NA & 0.86 & $\{1,2,3,5,6,7,11\}$ & 0.38 & $\{1,2,4,5,6,7,8\}$ \\
\hline M20-7.5 & $(1,1 / 7,1 / 21,0,0)$ & 0.00 & NA & 0.29 & $\{1,2,3,5,6,7,12\}$ & 0.00 & NA \\
\hline
\end{tabular}


Table 6

Comparisons of projection rankings

\begin{tabular}{llccllcc}
\hline Design & EV & Maxest & GMA & Design & EV & Maxest & GMA \\
\hline $20-5.1$ & $(1,1,1,0,0)$ & 1 & 1 & $20-5.6$ & $(1,1 / 5,4 / 10,0,0)$ & 3 & 4 \\
$20-5.2$ & $(1,2 / 5,3 / 10,0,0)$ & 2 & 6 & $20-5.7$ & $(1,1,1,0,0)$ & 1 & 3 \\
$20-5.3$ & $(1,1,1,0,0)$ & 1 & 5 & $20-5.8$ & $(1,0,0,0,0)$ & 5 & 9 \\
$20-5.4$ & $(1,1 / 5,2 / 10,0,0)$ & 4 & 7 & $20-5.9$ & $(1,1 / 5,2 / 10,0,0)$ & 4 & 8 \\
$20-5.5$ & $(1,1,1,0,0)$ & 1 & 2 & $20-5.10$ & $(1,1 / 5,2 / 10,0,0)$ & 4 & 10 \\
\hline
\end{tabular}

maxest, and MMA projections (Xu, 2003). For example, there are 10 classes of projections onto 4 three-level and 3 two-level factors in $O A\left(36,3^{12} 2^{11}\right)$. The maxest projection with columns 1 , $3,4,5,13,14$, and 15 has 3 clear main effects and 3 clear 2fi's. In contrast, the MMA projection with columns 1, 5, 9, 10, 16, 21, and 23 has no clear effects. Therefore, an MMA design may not be a maxest design.

Table 7

Projections of $O A\left(36,3^{12} 2^{11}\right)$

\begin{tabular}{llllll}
\hline$\left(n_{3}, n_{2}\right)$ & Class & Maxest & & MMA & \\
& & EV & Columns & EV & Columns \\
\hline$(1,1)$ & 1 & $(1,1,1)$ & $\{1,13\}$ & $(1,1,1)$ & $\{1,13\}$ \\
$(1,2)$ & 1 & $(1,1,1)$ & $\{1,13,14\}$ & $(1,1,1)$ & $\{1,13,14\}$ \\
$(1,3)$ & 1 & $(1,1,1)$ & $\{1,13,14,15\}$ & $(1,1,1)$ & $\{1,13,14,15\}$ \\
$(1,4)$ & 1 & $(1,1,1)$ & $\{1,13,14,15,16\}$ & $(1,1,1)$ & $\{1,13,14,15,16\}$ \\
$(2,1)$ & 2 & $(1,1,1)$ & $\{1,2,20\}$ & $(1,1,1)$ & $\{1,2,20\}$ \\
$(2,2)$ & 3 & $(1,1,1)$ & $\{1,3,15,16\}$ & $(1,1,1)$ & $\{1,3,15,16\}$ \\
$(2,3)$ & 8 & $(1,1,1)$ & $\{2,3,13,15,23\}$ & $(1,1,1)$ & $\{2,3,13,15,23\}$ \\
$(2,4)$ & 10 & $(1,4 / 8,18 / 26)$ & $\{1,7,13,14,15,16\}$ & $(1,4 / 8,16 / 26)$ & $\{1,3,15,16,19,20\}$ \\
$(3,1)$ & 6 & $(1,1,1)$ & $\{1,2,8,20\}$ & $(1,1,1)$ & $\{1,2,8,20\}$ \\
$(3,2)$ & 25 & $(1,1,1)$ & $\{4,9,10,16,21\}$ & $(1,1,1)$ & $\{4,9,10,16,21\}$ \\
$(3,3)$ & 32 & $(1,5 / 9,3 / 33)$ & $\{5,6,12,13,16,17\}$ & $(1,0,0)$ & $\{2,3,4,15,21,23\}$ \\
$(3,4)$ & 4 & $(1,0,6 / 42)$ & $\{8,10,12,13,14,15,20\}$ & $(1,0,0)$ & $\{2,3,4,13,15,21,23\}$ \\
$(4,1)$ & 16 & $(1,5 / 9,0)$ & $\{1,4,5,11,13\}$ & $(1,1 / 9,0)$ & $\{1,5,9,10,21\}$ \\
$(4,2)$ & 24 & $(1,3 / 10,0)$ & $\{1,2,10,11,14,21\}$ & $(1,2 / 10,0)$ & $\{1,5,9,10,16,21\}$ \\
$(4,3)$ & 10 & $(1,3 / 11,3 / 51)$ & $\{1,3,4,5,13,14,15\}$ & $(1,0,0)$ & $\{1,5,9,10,16,21,23\}$ \\
$(5,1)$ & 6 & $(1,3 / 11,0)$ & $\{1,2,3,4,6,13\}$ & $(1,0,0)$ & $\{1,2,6,7,11,21\}$ \\
$(5,2)$ & 5 & $(1,3 / 12,0)$ & $\{1,3,4,5,6,16,17\}$ & $(1,0,0)$ & $\{1,2,6,7,11,18,21\}$ \\
\hline
\end{tabular}

\section{Conclusions and Remarks}

This paper introduces a new criterion to address the problem of optimal factor assignment in factorial designs. The maxest criterion is an extension of the maximum resolution criterion in regular designs and is a refinement of Webb's resolution criterion for general factorial designs. It is coding-dependent, which differs from other coding-independent criteria such as GMA and MMA. 
The maxest criterion is applied to study projections of nonregular designs. Comparisons with other projective properties such as the geometric projection, hidden projection, GMA, and MMA projection are also discussed. The new classification is simpler and is oriented towards statistical modelling. The maxest criterion can also be applied for other purposes such as constructing new optimal designs for factor screening and response surface exploration. This will be studied later.

\section{Acknowledgements}

This paper forms part of the author's Ph.D. thesis in the Department of Statistics, the University of Michigan. The author is grateful to Professor C. F. Jeff Wu for his invaluable advice and help. The author also thanks Professor Rahul Mukerjee and two referees for their appreciative comments.

\section{References}

Box, G. E. P., Hunter, J. S., 1961. The $2^{k-p}$ fractional factorial designs. Technometrics 3, 311-351, 449-458.

Chen, H., Hedayat, A. S., 1998. $2^{n-m}$ designs with resolution III or IV containing clear two-factor interactions. J. Statist. Plann. Inference 75, 147-158.

Cheng, S. W., Wu, C. F. J., 2001. Factor screening and response surface exploration (with discussion). Statistica Sinica 11, 553-604.

Deng, L. Y., Li, Y., Tang, B., 2000. Catalogue of small runs nonregular designs from Hadamard matrices with generalized minimum aberration. Commun. Statist.-Theory Meth. 29, 1379-1395.

Deng, L. Y, Tang, B., 1999. Generalized resolution and minimum aberration criteria for Plackett-Burman and other nonregular factorial designs. Statistica Sinica 9, 1071-1082.

Fries, A., Hunter, W. G., 1980. Minimum aberration $2^{k-p}$ designs. Technometrics 22, 601-608.

Hamada, M., Wu, C. F. J., 1992. Analysis of designed experiments with complex aliasing. Journal of quality technology 24, 130-137.

Hedayat, A. S., Sloane, N. J. A., Stufken, J., 1999. Orthogonal arrays: Theory and Applications. Springer, New York.

Lin, D. K. L., Draper, N. R., 1995. Screening properties of certain two-level designs. Metrika 42, 99-118.

Milliken, G. A., 1971. New criterion for estimability for linear models. Ann. Math. Statist. 42, 1588-1594.

Rechtschaffner, R. L., 1967. Saturated fractions of $2^{n}$ and $3^{n}$ factorial designs. Technometrics $9,569-576$. 
Srivastava, J. N., Chopra, D. V., 1971. Balanced optimal $2^{m}$ fractional factorial designs of resolution $\mathrm{V}, m \leq 6$. Technometrics 13, 257-269.

Sun, D. X., Li, W., Ye, K. Q., 2001. An algorithm for sequentially constructing non-isomorphic orthogonal designs and its applications, SUNYSB Preprint, AMS-02-13.

Tang, B, Ma, F., Ingram, D., Wang, H., 2000. Bounds on the maximum numbers of clear two-factor interactions for $2^{m-P}$ designs of resolution III and IV. The Canadian Journal of Statistics 30, 127-136.

Wang, J. C., Wu, C. F. J., 1995. A hidden projection properties of Plackett-Burman and related designs. Statistica Sinica 5, 235-250.

Webb, S., 1968. Non-orthogonal designs of even resolution. Technometrics 10, 291-299.

Wu, C. F. J., Chen, Y., 1992. A graph-aided method for planning two-level experiments when certain interactions are important. Technometrics 34, 162-175.

Wu, C. F. J., Hamada, M., 2000. Experiments: Planning, Analysis and Parameter Design Optimization. New York, Wiley.

Wu, C. F. J., Zhu, Y., 2003. Optimal selection of single arrays for parameter design experiments. Statistica Sinica 13, 1179 -1200.

$\mathrm{Xu}$, H., 2003. Minimum moment aberration for nonregular designs and supersaturated designs. Statistica Sinica 13, 691-708.

$\mathrm{Xu}$, H., Wu, C. F. J., 2001. Generalized minimum aberration for asymmetrical fractional factorial designs. Ann. Statist. 29, 1066-1077. 
Appendix A. 20-run Hall's designs of types $\mathbf{N}$ and $\mathbf{P}$

\begin{tabular}{ccccccccccccccccccccc}
\multicolumn{11}{c}{ Type N } \\
\hline Run & 1 & 2 & 3 & 4 & 5 & 6 & 7 & 8 & 9 & 10 & 11 & 12 & 13 & 14 & 15 & 16 & 17 & 18 & 19 \\
\hline 1 & 1 & 1 & 1 & 1 & 1 & 1 & 1 & 0 & 0 & 0 & 0 & 0 & 0 & 0 & 0 & 0 & 0 & 0 & 0 \\
2 & 1 & 1 & 0 & 1 & 0 & 0 & 0 & 0 & 0 & 1 & 1 & 0 & 0 & 1 & 1 & 0 & 0 & 1 & 1 \\
3 & 1 & 1 & 0 & 0 & 1 & 0 & 0 & 1 & 1 & 0 & 0 & 1 & 1 & 0 & 0 & 0 & 0 & 1 & 1 \\
4 & 1 & 1 & 0 & 0 & 0 & 1 & 0 & 1 & 1 & 0 & 0 & 0 & 0 & 1 & 1 & 1 & 1 & 0 & 0 \\
5 & 1 & 1 & 0 & 0 & 0 & 0 & 1 & 0 & 0 & 1 & 1 & 1 & 1 & 0 & 0 & 1 & 1 & 0 & 0 \\
6 & 1 & 0 & 0 & 1 & 1 & 1 & 1 & 1 & 1 & 1 & 1 & 1 & 1 & 1 & 1 & 0 & 0 & 0 & 0 \\
7 & 1 & 0 & 1 & 1 & 1 & 0 & 0 & 1 & 0 & 1 & 0 & 1 & 0 & 0 & 1 & 1 & 1 & 1 & 0 \\
8 & 1 & 0 & 1 & 1 & 1 & 0 & 0 & 0 & 1 & 0 & 1 & 0 & 1 & 1 & 0 & 1 & 1 & 0 & 1 \\
9 & 1 & 0 & 1 & 0 & 0 & 1 & 1 & 1 & 0 & 1 & 0 & 0 & 1 & 1 & 0 & 1 & 0 & 1 & 1 \\
10 & 1 & 0 & 1 & 0 & 0 & 1 & 1 & 0 & 1 & 0 & 1 & 1 & 0 & 0 & 1 & 0 & 1 & 1 & 1 \\
11 & 0 & 1 & 0 & 1 & 1 & 1 & 1 & 1 & 1 & 1 & 1 & 0 & 0 & 0 & 0 & 1 & 1 & 1 & 1 \\
12 & 0 & 1 & 1 & 1 & 0 & 1 & 0 & 1 & 0 & 0 & 1 & 1 & 1 & 1 & 0 & 0 & 1 & 1 & 0 \\
13 & 0 & 1 & 1 & 1 & 0 & 1 & 0 & 0 & 1 & 1 & 0 & 1 & 1 & 0 & 1 & 1 & 0 & 0 & 1 \\
14 & 0 & 1 & 1 & 0 & 1 & 0 & 1 & 1 & 0 & 0 & 1 & 1 & 0 & 1 & 1 & 1 & 0 & 0 & 1 \\
15 & 0 & 1 & 1 & 0 & 1 & 0 & 1 & 0 & 1 & 1 & 0 & 0 & 1 & 1 & 1 & 0 & 1 & 1 & 0 \\
16 & 0 & 0 & 1 & 0 & 0 & 0 & 0 & 1 & 1 & 1 & 1 & 0 & 0 & 0 & 0 & 0 & 0 & 0 & 0 \\
17 & 0 & 0 & 0 & 1 & 0 & 0 & 1 & 1 & 0 & 0 & 0 & 0 & 1 & 0 & 1 & 0 & 1 & 0 & 1 \\
18 & 0 & 0 & 0 & 1 & 0 & 0 & 1 & 0 & 1 & 0 & 0 & 1 & 0 & 1 & 0 & 1 & 0 & 1 & 0 \\
19 & 0 & 0 & 0 & 0 & 1 & 1 & 0 & 0 & 0 & 1 & 0 & 1 & 0 & 1 & 0 & 0 & 1 & 0 & 1 \\
20 & 0 & 0 & 0 & 0 & 1 & 1 & 0 & 0 & 0 & 0 & 1 & 0 & 1 & 0 & 1 & 1 & 0 & 1 & 0
\end{tabular}

\begin{tabular}{cccccccccccccccccccc}
1 & 0 & 0 & 0 & 0 & 1 & 0 & 0 & 0 & 0 & 1 & 1 & 0 & 0 & 1 & 1 & 0 & 1 & 1 & 0 \\
2 & 0 & 1 & 1 & 1 & 1 & 0 & 1 & 1 & 1 & 0 & 0 & 0 & 1 & 1 & 1 & 0 & 1 & 0 & 0 \\
3 & 1 & 0 & 1 & 1 & 1 & 1 & 0 & 1 & 1 & 1 & 0 & 0 & 0 & 1 & 0 & 1 & 0 & 1 & 0 \\
4 & 1 & 1 & 0 & 1 & 1 & 1 & 1 & 0 & 1 & 1 & 1 & 0 & 0 & 0 & 0 & 0 & 1 & 0 & 1 \\
5 & 1 & 1 & 1 & 0 & 1 & 1 & 1 & 1 & 0 & 0 & 1 & 1 & 0 & 0 & 1 & 0 & 0 & 1 & 0 \\
6 & 0 & 0 & 0 & 0 & 0 & 1 & 1 & 1 & 1 & 1 & 0 & 1 & 1 & 0 & 0 & 0 & 1 & 1 & 0 \\
7 & 0 & 1 & 1 & 1 & 0 & 1 & 0 & 0 & 0 & 1 & 0 & 1 & 0 & 0 & 1 & 1 & 1 & 0 & 0 \\
8 & 1 & 0 & 1 & 1 & 0 & 0 & 1 & 0 & 0 & 0 & 1 & 0 & 1 & 0 & 0 & 1 & 1 & 1 & 0 \\
9 & 1 & 1 & 0 & 1 & 0 & 0 & 0 & 1 & 0 & 0 & 0 & 1 & 0 & 1 & 0 & 0 & 1 & 1 & 1 \\
10 & 1 & 1 & 1 & 0 & 0 & 0 & 0 & 0 & 1 & 1 & 0 & 0 & 1 & 0 & 1 & 0 & 0 & 1 & 1 \\
11 & 1 & 0 & 0 & 1 & 0 & 1 & 0 & 0 & 1 & 0 & 1 & 1 & 1 & 1 & 1 & 0 & 0 & 0 & 0 \\
12 & 1 & 1 & 0 & 0 & 1 & 0 & 1 & 0 & 0 & 1 & 0 & 1 & 1 & 1 & 0 & 1 & 0 & 0 & 0 \\
13 & 0 & 0 & 0 & 1 & 1 & 0 & 1 & 0 & 1 & 0 & 0 & 1 & 0 & 0 & 1 & 1 & 0 & 1 & 1 \\
14 & 1 & 0 & 0 & 0 & 1 & 1 & 0 & 1 & 0 & 0 & 0 & 0 & 1 & 0 & 1 & 1 & 1 & 0 & 1 \\
15 & 0 & 0 & 1 & 1 & 1 & 0 & 0 & 1 & 0 & 1 & 1 & 1 & 1 & 0 & 0 & 0 & 0 & 0 & 1 \\
16 & 0 & 1 & 1 & 0 & 1 & 1 & 0 & 0 & 1 & 0 & 1 & 1 & 1 & 1 & 0 & 1 & 1 & 1 & 1 \\
17 & 0 & 1 & 0 & 0 & 0 & 0 & 0 & 1 & 1 & 0 & 1 & 0 & 0 & 0 & 0 & 1 & 0 & 0 & 0 \\
18 & 0 & 1 & 0 & 1 & 0 & 1 & 1 & 1 & 0 & 1 & 1 & 0 & 1 & 1 & 1 & 1 & 0 & 1 & 1 \\
19 & 1 & 0 & 1 & 0 & 0 & 0 & 1 & 1 & 1 & 1 & 1 & 1 & 0 & 1 & 1 & 1 & 1 & 0 & 1 \\
20 & 0 & 0 & 1 & 0 & 0 & 1 & 1 & 0 & 0 & 0 & 0 & 0 & 0 & 1 & 0 & 0 & 0 & 0 & 1 \\
\hline
\end{tabular}

